\title{
Los modelos pedagógicos y su relación con las concepciones del derecho: puntos de encuentro con la educación en derecho
}

\section{Pedagogical models and their relationship with the conceptions of law: meeting points with education in law}

Álvaro Garzón-Saladen
Zilath Romero-González

Recibido: octubre 25 de 2017 Aceptado: diciembre 28 de 2017

\begin{abstract}
Resumen
El estudio de los modelos pedagógicos tiene relevancia en conexión con las concepciones del derecho, por cuanto los modelos deben estar de acuerdo con el derecho objeto del proceso educativo, con los sustentos teóricos del sistema jurídico y de la cultura jurídica. Este documento hace parte de las indagaciones teóricas con un énfasis hermenéutico, un diálogo de lo jurídico y lo pedagógico, con una visión transversal de la formación en derecho. La reflexión indica que los aportes realizados por la pedagogía jurídica admiten la existencia de un juego de argumentos, como estrategia para aprehender y re-aprender fenómenos del contexto jurídico, posibilitando una nueva conciencia de lo jurídico. Se concluye que la concepción pedagógica influye en la formación del abogado, de modo que el modelo pedagógico debe brindar una aplicabilidad y compenetración de la auscultación del ciudadano jurídico como profesional de derecho que influya en la realidad social.
\end{abstract}

Palabras clave: modelos pedagógicos, concepciones del derecho, positivismo, educación.

\begin{abstract}
The study of the pedagogical models have important connection to the views of law because they must be in line with the object of the educational process in law studies and the theories of both the law and its law culture. This paper is part of the theorethical surveys whose approach is hermeneutic, dialogic law and pedagogic has a whole view of the process of teaching and learning in law studies. The insights show that the contributions made by the law pedagogic admit the existence of game of arguments as strategies to learn and relearn the law context phenomena making posible a new awareness of what is law. To close it can be said that the pedagoic view influences the formation of the lawyer therefore the pedagogic model needs to offer the usage and the compenetration of the law citizen auscultation as profesional person in this field with an influecing power in the social reality.
\end{abstract}

Keywords: pedagogical models, conceptions of law, positivism, education.

1 Abogado, Magíster en Derecho, Estudiante Doctorado en Ciencias de la Educación, Universidad Libre, Cartagena, Colombia. E-mail: alvarogarzonsaladen@gmail.com

2 Administradora para el Desarrollo Regional, Doctora en Ciencias de la Educación, Universidad Libre, Cartagena, Colombia. E-mail: zilathromero@yahoo.com 


\section{Introducción}

Cuando se estudia derecho normalmente no se mira su transversalidad en relación con las concepciones del derecho mismo y los modelos pedagógicos. Es decir que quedan fuera de consideración cuestiones tan importantes como la manera en que puede influenciar la concepción del derecho sobre el modelo, o cómo puede resistirse el proceso educativo del derecho a un cambio que ponga en diálogo nociones como concepción jurídica y modelo pedagógico.

Los aportes realizados por la pedagogía del derecho propician la existencia de un juego de argumentos que funciona muy bien como estrategia para aprehender y re-aprender fenómenos del contexto jurídico, posibilitando una nueva conciencia de lo jurídico (Carbonell, 2012). La concepción pedagógica influye directamente en la formación del abogado, por lo que el modelo pedagógico debe orientarse hacia la formación de ciudadanos jurídicos que, en su condición de profesionales del derecho, apliquen sus habilidades a la transformación de la realidad social, al igual que ocurre en otras profesiones (Lago-de Vergara, Gamoba-Suárez \& Montes-Miranda, 2014).

En este sentido, se justifica auscultar estos terrenos desde la transversalidad para mejor entender la realidad y la crisis de la formación de los estudiantes de derecho, tal y como se hace en otras disciplinas de carácter sociohumanístico (Silva-Cañaveral, 2016). Las dimensiones cualitativas, bajo las cuales se aborda la presente reflexión, aportan la comprensión de razones, lógicas, racionalidades, visiones, modos de ser y de comportarse, que llenan el dato de contenido y permiten, desde los múltiples actores sociales, conocer la diversidad y heterogeneidad social (Galeano-Marín, 2016).

Este documento hace parte de las indagaciones teóricas con un énfasis hermenéutico, un diálogo de lo jurídico y lo pedagógico, con una visión transversal de la formación en derecho. En una segunda fase se realizará la investigación de campo sobre los modelos pedagógicos que se reflejan en el proceso de formación de los estudiantes de derecho de la Universidad Libre.

En un esfuerzo por abarcar los puntos centrales de esta discusión, el texto inicia presentando una amplia definición de modelos pedagógicos, antes de señalar los puntos de encuentro entre dichos modelos y la educación en derecho. Finalmente, se presentan conclusiones e inconclusiones proponiendo un diálogo intersubjetivo de diferentes autores, con el fin de generar choques ideológicos y nueva aprehensión sobre lo explorado en cuanto a la educación en derecho.

\section{Los modelos pedagógicos y su finalidad}

La palabra modelo está ligada, en su origen griego, a distintas acepciones. Su significado se vincula, por igual, con las actuales nociones de prototi-

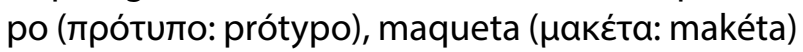
y metro o medida ( $\mu$ ćtрo: métro) (Sanakirja.org, 2016). Por modelo también se entiende algo que media entre los aspectos característicos de una teoría y la praxis. Ampliando la conceptualización a partir de esto, puede afirmarse que un modelo es una herramienta que se utiliza para describir, decidir e interpretar actitudes en diferentes situaciones, haciendo viable el reproducir hipótesis y elaborar estrategias para su intervención.

En el contexto de este trabajo, conviene resaltar la relación existente entre el griego $\mu \varepsilon \dot{t} \rho o$ (métro) y el latín modus (manera o medida), que permite entender el concepto de modelo como iniciador o indicador de algo. Este enfoque deja como síntesis un modelo que abstrae los temas relevantes sobre un problema a resolver, de entre un sinnúmero de detalles en los cuales el problema puede estar sumergido. Representa la estructura del problema, su forma, ya que cada problema tiene una forma, y a menudo se dificulta entenderlo con claridad suficiente para describir esa estructura (Gómez, 2008). 
En cuanto a la pedagogía, Durkheim, citado por Zambrano (2017), la asume como la teoría práctica de la educación, basada en el acompañamiento que el adulto realiza en favor de la infancia. Es la primera guianza retomada y arrastrada desde el pensamiento griego a través del paidotriba, el instructor de los niños atenienses. En este sentido, la pedagogía es el arte de enseñar, una teoría práctica de la educación. Por su parte, Vygotsky, citado por De Zubiría-Samper (2002), indica que esta no debe orientarse hacia el ayer sino hacia el mañana del desarrollo infantil; hacer lo contrario sería orientarse por la línea de menor resistencia, atendiendo a la debilidad del niño y no a su fuerza.

Sin embargo, la conceptualización de la pedagogía no solo se puede circunscribir en el marco de las ciencias de la educación, pues también hace referencia al ideal del hombre asignado por el eco de la sociología francesa en la búsqueda del nuevo hombre (Fernández-Guerrero \& González-Ferro, 2017). Así, la pedagogía se relaciona con la antropología, el derecho, la filología, la filosofía, la psicología y la política, como una forma que pretende dialogar respetando el otro (Santos, 2011). El modelo pedagógico representa, pues, el ideal de hombre y de mujer que se proyecta para un contexto histórico, social y cultural determinado, en coherencia con un ideal de hombre y de mujer proyectado para el contexto universal (Díaz-Monsalve \& Quiroz-Posada, 2001).

En este sentido, un modelo pedagógico es un sistema formal que busca interrelacionar los agentes básicos de la comunidad educativa con el conocimiento científico, para conservarlo, producirlo o recrearlo dentro de un contexto histórico, geográfico y cultural determinado (García-Quiroga, Coronado \& Giraldo-Ospina, 2017). Además, el modelo pedagógico debe responder a una realidad socio-político-económica, la cual debe tener como garante la reestructuración en la puesta en marcha del mismo, en relación a su carácter holístico (Espino-de Lara, 2015).
Las siguientes preguntas: ¿qué enseñar?, ¿cómo enseñar?, ¿qué y cómo evaluar?, son importantes para el desarrollo de la construcción del modelo pedagógico, en cuanto permiten indicar los contenidos, exteriorizar la forma de transmitirlos y revelar el nivel de logro de los objetivos de formación que se hayan propuesto en torno a ellos. Además de las anteriores preguntas, el modelo lleva implícitos algunos cuestionamientos que ponen de relieve los propósitos educativos (¿para qué enseñar?) y formulan juicios de valor acerca de los fenómenos abordados en el proceso de formación (¿cómo reflexionar sobre lo estudiado?).

Para sustentar este planteamiento, se debe recordar a Not, citado por Arcila (2013), quien indica que, de acuerdo con el enfoque que se dé a la relación entre aprendiz y enseñante, existen tres métodos para estructurar un modelo. En primer lugar se encuentra el método autestructural, que reconoce al niño como el verdadero constructor de su aprendizaje. En segundo término está la heteroestructuración, que, tras asumir que el conocimiento se realiza fuera del salón de clase, limita la labor de la escuela a transmitir la cultura humana. En tercer lugar, y como síntesis de los dos anteriores, está el método interestructurante, que entiende la educación como una labor compartida entre enseñante y aprendiz.

Porlán, citado por Horsori (2002), establece que todo modelo pedagógico, para ser considerado como tal, requiere de un enfoque, una metodología y unas formas de evaluación; precisados estos tres elementos, es además necesario identificar la percepción que cada modelo tiene del docente, del alumno y de los saberes que enseña. Así mismo, Flórez, citado por Ortíz-Ocaña (2013), define el modelo pedagógico como la representación de las relaciones que predominan en el acto de enseñar; de acuerdo con este autor, se trata también de un paradigma que puede coexistir con otros y que sirve para organizar la búsqueda de nuevos conocimientos en el campo de la pedagogía. 
De Zubiría-Samper (2002) indica que el modelo debe fortalecer el favorecimiento de las operaciones de análisis, la formación de un pensamiento sistémico y global y el desarrollo de la habilidad para trabajar cooperativamente con los compañeros. Recientemente Nussbaum, citada por Guichot-Reina (2015), en su discurso pronunciado al recibir el premio Príncipe de Asturias de ciencias sociales, basado en el enfoque de las capacidades, presentó algunos lineamientos que debería seguir la educación: la de contribuir a formar el pensamiento crítico y reflexivo y la de ser pluralista y formarnos en una ciudadanía múltiple o compleja. Es decir, los modelos pedagógicos deberán favorecer el desarrollo de competencias en cuanto al uso y apropiación de tecnología, la comprensión lectora, las competencias matemáticas básicas y la habilidad para resolver problemas del contexto (Barrera-Mesa, Fernández-Morales \& Duarte, 2017; López-de Parra, Polanco-Perdomo \& Correa-Cruz, 2017).

\section{Puntos de encuentro entre los modelos pe- dagógicos y la educación en derecho}

\subsection{Concepciones del derecho}

No pueden sobrevenir modificaciones en el pensamiento humano si no se invita a una crítica constante, sin sesgos que universalicen las que el historiador alemán Koselleck denomina "verdades occidentales". En el ámbito que concierne a este estudio, los saltos cualitativos solo se pueden realizar si se comprende la realidad de lo que se estudia en el campo del derecho (Cortina, 1999).

Para empezar, es necesario tener en cuenta que, en el marco doctrinal del derecho, se encuentran dos corrientes de pensamiento opuestas: el iusnaturalismo y el positivismo jurídico. Vinculadas, respectivamente, a la fenomenología y la hermenéutica jurídica, y en tanto puntos de referencia para conceptualizar nociones como ser humano, persona o ciudadano (Zúñiga, 2016), representan la base a partir de la cual se definen las estructuras mismas del campo jurídico y la cultura jurídica, y por tanto la forma como debe abordarse su estudio y enseñanza.

El iusnaturalismo o derecho natural asume que en la base de la dignidad humana subyace una intuición moral básica. A diferencia de las utopías sociales de la felicidad, el derecho natural se orienta a la pretensión, a la protección de la dignidad humana; esta es una teoría mínima del reconocimiento ligado a lo moral y a la dignidad del ser humano (Guastini, 1998).

En la misma vía, Ferrajoli (1999) indica que son derechos fundamentales todos aquellos derechos subjetivos que corresponden universalmente a todos los seres humanos en cuanto a dotados del status de personas, de ciudadanos, o de personas capaces de obrar; entendiendo por "derecho subjetivo" cualquier expectativa positiva (de prestaciones) o negativa (de no sufrir lesiones) adscrita a un sujeto por una norma jurídica; y por "status" la condición del sujeto, prevista asimismo por una norma jurídica positiva, como presupuesto de su idoneidad para ser titular de situaciones jurídicas o autor de los actos que son de estas.

Por su parte, el positivismo solo reconoce como derecho aquello que está contemplado en un ordenamiento jurídico concreto; es decir, la validez de la norma solo se da en la medida en que está por escrito y se encuentra vigente en un país, durante determinado momento histórico. En otros términos, lo que en derecho se conoce como ley positiva. De acuerdo con esto, es posible encontrarse ante distintas realidades o contextos $y$, por tanto, frente a diferentes formulaciones e interpretaciones de la norma. A la luz de este paradigma, la norma resulta vital para la construcción de las dinámicas sociales y de lo que de estas se desprende. En consecuencia, al estar en la base de la realidad social, la norma se debe modificar de forma positiva, propositiva, reflexiva, de acuerdo con las modificaciones que sufra la sociedad que en ella se sostiene. 
En este sentido, el derecho tiene un carácter evolutivo. Sufre las constantes metamorfosis que le imprimen el periodo histórico, los vaivenes de la política, el desarrollo socioeconómico, las guerras o las diferencias asimétricas en la forma como se administra la propiedad privada. En otras palabras, el derecho evoluciona a partir de las formas como avanza la humanidad.

En cuanto al positivismo jurídico, Carrillo (2008) plantea que este puede subsumirse en el principio de sujeción a la ley, enmarcado en la abdicación valorativa del jurista, el argumento del legislador racional, la subordinación del jurista a la ley, y el principio de justicia del derecho, que entiende el derecho como un sistema. El positivismo justifica la tesis anterior valiéndose de los siguientes principios:

i) Todo el derecho está contenido en la ley y, por tanto, es la única fuente del derecho. En todo caso, el derecho positivo es la fuente más importante, mientras que el resto de criterios son meramente subsidiarios o auxiliares.

ii) Los juristas deben usar el derecho positivo vigente tal y como ha sido sancionado por el legislador, evitando introducir valoraciones particulares o que no hayan sido previstas por el legislador.

Carrillo (2008), citando a Calsamiglia, extiende los argumentos anteriores presuponiendo una visión conservadora de la función judicial y doctrinaria del derecho. Según esto, los juristas solo deben dedicarse a transcribir o reproducir, acríticamente, un cuerpo de reglas, dejando a un lado el papel innovador y de adaptación del derecho de las realidades cambiantes y dinámicas. El sujeto, el supuesto de sujeción de la ley, por lo demás, ataca a la autonomía política del jurista tratando de restringir la actividad económica creadora del derecho mismo, y erige la seguridad jurídica como valor más importante del ordenamiento jurídico, inclusive por encima de la justicia.
Esto trae a colación el debate entre Hart y Dworking. El primero indica que, en caso de lagunas en el ordenamiento jurídico, el juez debe apelar a su discrecionalidad. El segundo infiere lo contrario: señala que el juez debe hallar la solución en el sistema jurídico, entendiendo que este no solo está compuesto por reglas positivas sino que incluye además principios (morales) y directrices (políticas). De allí que, para Dworking, el principio de sujeción a la ley deba sustituirse por el principio de sujeción al derecho. Sin embargo, este debate es mucho más amplio, en la medida en que se centra sobre la regla del reconocimiento, la existencia de reglas y principios en la teoría y la práctica jurídica, y el problema de la discrecionalidad de los jueces en los casos difíciles. Esto, en consecuencia, implica discutir en torno al poder creador del juez, los métodos de interpretación y la posibilidad de haIlar respuestas correctas en el derecho (Rodríguez, 2002).

Con el propósito de ofrecer un aporte desde lo teórico-crítico al esclarecimiento de estas cuestiones, nos podemos apoyar en Ferrajoli, quien indica que el actual derecho no solo programa sus "formas de producción a través de normas de procedimiento sobre la formación de leyes y demás disposiciones", sino también sus "contenidos sustanciales, vinculados normativamente a los principios y a los valores inscritos en sus constituciones, mediante técnicas de garantía". Lo anterior representa la alteración del modelo positivista clásico en distintos planos, a saber:

i) en el plano de la teoría del derecho supone una revisión de la teoría de la validez y vigencia y en una nueva relación entre forma y sustancia de las decisiones; ii) en el plano de la teoría política, donde comporta una relación entre la concepción puramente procedimental de la democracia y el reconocimiento también de una dimensión sustancial; iii) en el plano de la teoría de la interpretación y de la aplicación de la ley, al incorporar una redefinición del papel del juez y una revisión de las formas y las condiciones de su sujeción a la ley; iv) el plano de la metateoría del derecho, y, por tanto, 
del papel de la ciencia jurídica, que resulta investida de una función no solamente descriptiva, sino crítica y proyectiva en relación con su objeto (Ferrajoli, 1999).

Según se puede ver, se trata de una dura crítica de Ferrajoli al positivismo y la forma en que se aplica, pues señala como inevitable la necesidad de que el juez pueda ser crítico ante el contenido de la norma. De lo anterior se deriva la correlación entre la interpretación del juez, o la hermenéutica jurídica, como sustento del aprendizaje del derecho, y las objeciones que plantea la fenomenología de lo jurídico, ambos pilares inmanentes de la ciencia jurídica y de los currículos de estudio de las escuelas o facultades del derecho en Colombia.

En este punto conviene resaltar el hecho de que esta es una realidad sumamente compleja en el caso de la sociedad colombiana. En la medida en que el nombramiento de los jueces suele estar condicionado por la relación entre la rama judicial y los sectores políticos y económicos, sus decisiones tienden a estar determinadas por factores externos que van más allá de una particular interpretación de la ley o una concepción específica del derecho (Sánchez, 2013). Podría decirse, incluso, que cualquier consideración por parte de las universidades acerca del jurista que les corresponde formar debe pasar por una seria exploración de esta realidad.

\subsection{Educación en derecho}

Las teorías sobre modelos pedagógicos han surgido como respuestas contradictoras o como forma complementaria. Un modelo transmisor y pasivo del educando genera una respuesta activista con una liberalidad en lo que debe ser o no objeto de aprendizaje. Las concepciones de los modelos y de la forma de ver el derecho parten de un problema epistemológico que se comunica tanto teóricamente como en la praxis.

En una concepción positivista ortodoxa, según la cual el derecho es el escrito $y$, por tanto, se ofrece culto a la ley, se confunde la legalidad con la justicia y las fuentes de derecho se jerarquizan, dándose mayor relevancia a las fuentes formales que a la realidad social. Un sistema jurídico que se centra en la legitimidad como un proceso democrático instrumentalizado en la creación de la ley, sesga la posibilidad de emancipar desde el derecho como forma de conciencia del individuo. En este sentido, el formalismo jurídico imperante en nuestra historia jurídica encuentra terreno para que el proceso de formación del jurista se identifique teóricamente con un modelo centrado en los códigos, con repetición acrítica y con inmovilidad conceptual a espaldas del contexto.

El positivismo ortodoxo genera resistencia teórica para estudiar el derecho desde los fenómenos para solo centrarse en lo clásico, al extremo de adorar las instituciones como estatuas sagradas que no pueden ser apreciadas desde ciertas perspectivas. Esa resistencia teórica mantiene la educación del derecho encerrada entre aulas, sin permitir la desobediencia a la conceptualización dogmática; de hecho, y solo por pensar un ejemplo, uno podría preguntarse si hoy la voluntad realmente es parte trascendental en los contratos de adhesión.

Por el contrario, un derecho crítico que parta de la realidad como fuente de la legitimidad y validez del derecho, no se funda en procesos formales $y$, en consecuencia, resulta incompatible con un modelo pedagógico clásico que se olvida del fenómeno social. Al asumir esta otra postura, se entiende que el derecho no es el sistema jurídico aislado, sino que engloba también el sistema jurídico, la cultura, los valores, los principios, la historia, la política, lo prehermenéutico y lo hermenéutico, donde no hay jerarquía de fuentes. Tener en cuenta la realidad y su jurisprudencia catalizadora, y asumir que tiene tanta trascendencia como la ley, hace que el modelo no solo se dedique a las fuentes formales, sino que la formación se extienda también a la comunidad.

Para explicar mejor esta dicotomía, se puede traer a colación el tema de la justicia transicional que 
se gestó alrededor del proceso de paz llevado a cabo entre el gobierno colombiano y las FARC. Este conjunto especial de medidas judiciales y políticas tendientes a dar tratamiento a casos de violación masiva de derechos humanos, no puede ser comprendido desde un derecho positivo ortodoxo. Este último se sustenta en conceptos que obedecen a una teoría normativista con afán de objetividad y aspiraciones de aceptación de conocimiento científico. En esta medida, su validación no permite la aplicación de valores o principios morales y se resiste a los controles constitucionales y convencionales sobre derechos humanos, que por su propia esencia no pueden ser condensados en reglas técnicas sino en normas abiertas que, vía interpretación, generan normas adscritas a la constitución o a las convenciones.

Desde la perspectiva del iusnaturalismo, en cambio, aunque se respeta la ley, se atiende también a principios y valores que, desde una mirada profunda a la realidad humana, respaldan los esfuerzos por basar la teoría jurídica sobre el estudio de la forma como se pueden producir decisiones justas. En otros términos, en este otro lado de la balanza se plantea la utilización del test de igualdad, la ponderación la razonabilidad y racionalidad, como parte del esfuerzo para fortalecer el iusnaturalismo e incluso una visión positivista suave que acepte lo expresado, con pretensión de mejorar en la toma de decisiones.

Si se extrapola lo antes dicho a un plano de formación, se entiende entonces la conveniencia de estudiar el derecho de forma transversal frente a la economía, la política, la filosofía, la pedagogía, etc. En lugar de partir de una teoría pura, por el contrario se tendría que plantear un derecho anidado en las ciencias sociales. Un modelo pedagógico así pensado debe apuntar a la transversalidad, donde las asignaturas no pueden mirarse como subconjuntos del gran conjunto universal sin intersecciones. Se debe entender en todo momento que la tendencia al ostracismo desconecta las redes de los conceptos, negando la posibilidad de una mayor comprensión.
Además, esta mirada holística debe asumir también que el abogado de hoy y del mañana está inmerso en entornos informáticos. Por supuesto, el uso de las TIC en la educación jurídica no asegura la aplicación de un modelo pedagógico que responda al contexto y a una metodología más activa que tienda el reconocimiento y conciencia de ser sujeto, objeto y otro en el proceso educativo. Si el discurso pedagógico, no obstante las TIC, genera un educando pasivo, que se evalúa a partir de una educación repetitiva, quiere decir que tal discurso sigue participando de un modelo clásico o tradicional. Esto implica que el manejo de las TIC debe implementarse desde una actitud y una aptitud críticas, cercanas al contexto que proponen estos sistemas de información.

En todo caso, el modelo y su concepción deben ser adaptables. Es claro que en la realidad de las sociedades existen problemas jurídicos que pueden ser resueltos desde una visión positivista ortodoxa o suave, mientras que otros demandan la mirada del iusnaturalismo. Así, por ejemplo, si la temática gira alrededor de una regla técnica o sus valores, se abre una concepción garantista de derechos fundamentales cuando se presenta laguna; tal es el caso del conflicto que se da entre el derecho a la eutanasia y el derecho a la vida. Este problema no lo resuelve una regla técnica, sino que depende de cada caso; por metodología, es apropiado que tal discusión se depure vía jurisprudencia, lo cual permite la viabilidad de adaptación del derecho.

En tal adaptabilidad, el currículo constituye una orientación frente a puntos neurálgicos o núcleos, acordes al contexto y al abogado que se quiere formar. Dicha orientación debe pensarse en respuesta a la necesidad de dotar al futuro abogado de herramientas de juicio imprescindibles para hacer frente a esos casos de la vida cotidiana (el desplazamiento de indígenas, la publicidad engañosa o la responsabilidad del Estado) cuya complejidad aumenta por el hecho de ser difundidos, muchas veces sin control, a través de las noticias que se difunden en noticieros, periódicos y redes sociales. 
En este sentido, la universidad debe ser muy sensible y estar muy atenta frente a estas problemáticas. Por ejemplo, la temática de la jurisdicción indígena ofrece un núcleo problémico interesante, por medio del cual se podrían desarrollar distintos temas; a modo de ejemplo: la jurisdicción y competencia, límite de la jurisdicción ordinaria indígena y contenciosa administrativa, los crímenes de lesa humanidad y el problema de la caducidad, por mencionar algunos. Esta clase de vivencias y de hechos notorios son provocativos para motivar el estudio como un laboratorio jurídico, rompiendo una concepción memorística y sin significado de la educación.

Un buen modelo pedagógico debe instar a la intervención del derecho para transformar en la extensión social y en la investigación. La transformación es importante incluso en el barrio, el caso más insignificante, por cuanto el abogado en tal modelo no solo es un solucionador del problema jurídico, sino que es transformador de lo jurídico. En lo social, el modelo pedagógico debe ser creador o catalizador de líderes para que el derecho se tenga como mecanismo de garantía. Así, para seguir con el ejemplo anterior, al indígena se le capacita solucionando su problema de jurisdicción. La invasión de su territorio por parte de la autoridad hegemónica, la soluciona el abogado que ejerce la contradicción vía constitucional; sin embargo, si tal solución no trasciende hacia el empoderamiento de la autonomía administrativa, judicial y legislativa, en este caso el derecho no libera, no emancipa. Por tal razón, el modelo pedagógico debe partir de que el derecho no es solo para juristas sino para la sociedad.

\section{Conclusiones}

La humanización de la vida debe penetrar el proceso de formación de los estudiantes de derecho. El derecho necesita un enramado que se dirija hacia la literatura, el cine, la poesía, la fábula y los ideales románticos. El abogado debe ser nutrido de sensibilidad y no puede ser visto como un computador o procesador de información, que arroja soluciones alejadas de las necesidades humanas del contexto en que se mueve.

Las concepciones pedagógicas y del derecho no pueden partir de posiciones absolutistas, pretendiendo sostener una epistemología que cae en el error de establecer el objeto de estudio como un ente inamovible cuando en realidad el cosmos cambia. Es insostenible aplicar, desde una lógica formal, el principio de identidad como objeto de estudio estático. La vida nos muestra que las teorías cambian y se contradicen. Ese diálogo debe conducir a una epistemología robustecida con la serenidad de que lo absoluto no puede ser aplicado a la sociedad, que es dinámica, ni al conocimiento, que es dogmático, dubitativo, progresivo, contradictorio. Esto nos hace reflexionar y entender que toda concepción de la educación debe ser abierta y ofrecer la oportunidad de construir y deconstruir desde un panorama dominado por lo holístico y lo transversal. Los modelos y las concepciones que propongamos deben ser acordes con la materia que estudiamos y estar abiertos a la transformación y la continua evaluación. El camino no es la radicalidad, sino la dialéctica.

\section{Referencias}

Arcila, J. F. (2013). Análisis de los modelos pedagógicos implementados en el sistema educativo no oficial del municipio de Santiago de Cali. Palmira, Colombia: Universidad Nacional De Colombia.

Barrera-Mesa, M., Fernández-Morales, F. H., \& Duarte, J. E. (2017). Aprendizaje basado en proyectos colaborativos mediados por TIC para el desarrollo de competencias en estadística. Saber, Ciencia y Libertad, 12 (2), 220-232. Recuperado de: http:// www.sabercienciaylibertad.org/ojs/index.php/ scyl/article/view/247

Carbonell, M. (2012). La enseñanza del derecho. México: Porrúa. 
Carrillo, Y. (2008). Problemas de las Ciencias Sociales y la Ciencia Jurídica. Cartagena, Colombia: Casa Editorial.

Cortina, A. (1999). Estudio preliminar a kant: metafísica de las costumbres. Madrid, España: Tecnos.

De Zubiría-Samper, J. (2002). Tratado de pedagogia conceptual - Los Modelos pedagógicos. Bogotá, Colombia: Fundación Alberto Merani.

Díaz-Monsalve, A. E., \& Quiroz-Posada, R. E. (2001). Corrientes pedagógicas, modelos pedagógicos y enfoques curriculares: relación sistemática entre ellos. Revista Avanzada, 10, 116-130.

Espino-de Lara, R. (2015). Educación holista. Revista Iberoamericana de Educación, 1-9. Recuperado de: https://rieoei.org/historico/deloslectores/330Espino.pdf

Ferrajoli, L. (1999). Derechos y garantías. La ley del mas débil. Madrid, España: Trotta.

Galeano-Marín, M. E. (2016). Diseño de proyectos en la investigación cualitativa. Medellín, Colombia: Universidad EAFIT.

García-Quiroga, B., Coronado, A., \& Giraldo-Ospina, A. (2017). Implementación de un modelo teórico a Priori de competencia matemática asociado al aprendizaje de un objeto matemático. Revista de Investigación, Desarrollo e Innovación, 7 (2), 301-315. doi: https://doi.org/10.19053/20278306. v7.n2.2017.6072

Gómez, M. (2008). Estilos de enseñanza y modelos pedagógicos. Bogotá, Colombia: Universidad de La Salle.

Guastini, R. (1998). Teoria e digmatica delle fonti. Milán, Italia: Giuffre.

Guichot-Reina, V. (2015). El enfoque de las capacidades de Martha Nussbaum y sus consecuencias educativas: hacia una pedagogía socrática y pluralista. Teoría de la Educación. Revista Interuniversitaria, 27 (2), 45-70. Recuperado de: http://revistas. usal.es/index.php/1130-3743/article/view/13799

Horsori. (2002). Enseñar y aprender. Bogotá, Colombia: Norma.

Lago-de Vergara, D., Gamoba-Suárez, A. A., \& Montes-Miranda, A. J. (2014). Calidad de la educación superior: un análisis de sus principales determinantes. Saber, Ciencia y Libertad, 9 (1), 157-170. doi: http://dx.doi.org/10.22525/sabcliber.2014v9n1.157170

Lopéz-de Parra, L., Polanco-Perdomo, V., \& Correa-Cruz, L. (2017). Mirada a las investigaciones sobre formación investigativa en la universidad latinoamericana: estado del arte 2010 a 2017. Revista de Investigación, Desarrollo e Innovación, 8 (1), 77-95. doi: https://doi.org/10.19053/20278306. v8.n1.2017.7371

Ortíz-Ocaña, A. (2013). Modelos pedagógicos y teorías del aprendizaje. Bogotá, Colombia: Ediciones de la U.

Rodríguez, C. (2002). La decisión judicial, El debate Hart-Dworking. Bogotá, Colombia: Siglo del Hombre Editores.

Sanakirja.org. (2016). Diccionario griego en línea. Recuperado de: http://webtionnaire.fr/search. php? $q=$ modelo $\& \mathrm{l}=4 \& \mid 2=7$

Sánchez, N. V. (2013). La crisis de la justicia en Colombia. Revista Cultural Unilibre.

Santos, B. S. (2011). Epistemologies du Sud. París, Francia: Editions de I’EHESS.

Silva-Cañaveral, S. (2016). La investigación-creación en el contexto de la formación doctoral en diseño y creación en Colombia. Revista de Investi- 
gación, Desarrollo e Innovación, 7 (1), 49-61. doi: https://doi.org/10.19053/20278306.v7.n1.2016.5601

Zambrano, A. (2017). Filosofía de la educación y de la pedagogía. Córdoba, Argentina: Editorial Brujas.

Zúñiga, L. (2016). Democracia, teoría crítica y ciudadanía. Cartagena, Colombia: Pluma de Mompox. 